\title{
X-ray multiphoton ionization dynamics of a water molecule irradiated by an x-ray free-electron laser pulse
}

\author{
Ludger Inhester, ${ }^{1,2, *}$ Kota Hanasaki, ${ }^{1,2, \dagger}$ Yajiang Hao (郝亚江), ${ }^{1,3,2, \ddagger}$ Sang-Kil Son (손상길), ${ }^{1,2, \S}$ and Robin Santra ${ }^{1,2,4, \|}$ \\ ${ }^{1}$ Center for Free-Electron Laser Science, DESY, Notkestrasse 85, 22607 Hamburg, Germany \\ ${ }^{2}$ The Hamburg Centre for Ultrafast Imaging, Luruper Chaussee 149, 22761 Hamburg, Germany \\ ${ }^{3}$ Department of Physics, University of Science and Technology Beijing, Beijing 100083, People's Republic of China \\ ${ }^{4}$ Department of Physics, University of Hamburg, Jungiusstrasse 9, 20355 Hamburg, Germany
}

(Received 8 July 2016; published 30 August 2016)

\begin{abstract}
We present a theoretical investigation of $\mathrm{x}$-ray multiphoton ionization dynamics of polyatomic molecules, based on the rate equation model and molecular electronic structure calculations. An efficient numerical procedure is developed to calculate photoionization cross sections, Auger rates, and fluorescence rates for all possible electronic multiple-hole configurations of molecules. We investigate the charge-state distribution of a water molecule after interaction with an intense $\mathrm{x}$-ray pulse and discuss its dependence on the fluence and the pulse duration of the $\mathrm{x}$-ray beam. Our results demonstrate that a water molecule exposed to an intense $\mathrm{x}$-ray pulse is more ionized than what would be expected within the independent-atom picture.
\end{abstract}

DOI: 10.1103/PhysRevA.94.023422

\section{INTRODUCTION}

New $\mathrm{x}$-ray light sources- $\mathrm{x}$-ray free-electron lasers (XFELs) [1] —hold the promise to allow for new methods in molecular structure determination [2,3]. Since smaller crystalline samples give rise to a lower diffraction signal, more intense x-ray light allows one to investigate smaller crystals and prospectively even noncrystalline samples [4]. Intense $\mathrm{x}$-ray light, however, causes inevitable radiation damage in the sample leading to a loss of its structural integrity and thus biasing the recorded signal. To overcome this limitation, diffraction-before-destruction strategies have been introduced $[4,5]$. Here the diffraction signal from a series of identical samples is accumulated. The individual samples are exposed to the $\mathrm{x}$-ray light during a very short pulse duration, such that the diffraction pattern is recorded before the molecular structure is altered as a result of radiation damage. To understand whether this concept is feasible also for noncrystalline samples, detailed understanding of the radiation damage is required.

The primary electronic process to be considered in the context of x-ray-matter interaction is ionization of core shell electrons. After a core electron has been ionized from the sample, the electronic structure relaxes typically via the Auger decay, in which the core hole is refilled by a valence electron and another valence electron, the Auger electron, is ionized and carries away the excess energy. With the unprecedentedly high fluence provided by XFELs, a sample may easily undergo these processes many times during an X-ray pulse, ejecting many electrons. These multiphoton multiple ionization dynamics can be understood as a sequence of consecutive photoionization and Auger decay processes, which has been verified by a series of gas-phase experiments of isolated atoms and molecules at LCLS and SACLA [6-13]. Eventually, for the molecular case,

\footnotetext{
*ludger.inhester@cfel.de

†kota.hanasaki@cfel.de

${ }^{\ddagger}$ haoyj@ustb.edu.cn

§sangkil.son@cfel.de

"robin.santra@cfel.de
}

the highly charged sample will start to dissociate by Coulomb explosion.

Many efforts have been devoted to the different aspects of radiation damage in coherent diffractive imaging of single particles. Pioneered by the work of Neutze et al. [4], radiation damage in samples was studied in terms of molecular dynamics simulation [14-16] or hydrodynamic expansion models $[17,18]$. These works are mainly based on an independent atom model to describe the electronic radiation damage. In this context electronic transition rates, i.e., photoionization cross sections and decay rates, were derived from the values for individual atoms. However, it has been found that relevant aspects of the radiation damage in molecules must be understood rather from the molecular electronic structure [7,19-23]. Specifically, a core ionization that is purely located on one atomic site may give rise to charging up at another atomic site of the molecule, so the charges are redistributed among neighboring atoms within the molecule. This charge rearrangement might yield different ion fragments than one would expect from the $\mathrm{x}$-ray ionization of the individual constituent atoms [20,22,23]. Theoretical descriptions for these observations have been developed so far only based on phenomenological models [19,24,25]. Recently we have discovered yet another molecular effect on ionization dynamics at high $\mathrm{x}$-ray intensity: The total charge for a molecule is higher than what one would expect if the molecule is treated as a collection of individual atoms [26]. In light of these findings, the development of $a b$ initio description of the $\mathrm{x}$-ray ionization dynamics of molecules is crucial.

In this work, we present a theoretical framework to describe $\mathrm{x}$-ray multiphoton multiple ionization dynamics of polyatomic molecules. We extend the XMOLECULE toolkit [27] to efficiently calculate photoionization cross sections and decay rates, based on molecular electronic structure calculations within XMOLECULE, and to solve a set of coupled rate equations to simulate ionization dynamics. The computational tool is versatile, so it is applicable to arbitrarily shaped molecules composed of any atomic species. The present method is applied to describe molecular effects on $\mathrm{x}$-ray multiphoton multiple ionization dynamics of a water molecule. We consider $\mathrm{H}_{2} \mathrm{O}$ 
because it is one of the simplest polyatomic molecules and it is relevant for x-ray radiation damage of biological systems.

The outline of this paper is as follows: Sec. II describes the computational methods used in this work. In Sec. III, we compare calculated molecular Auger rates with previous results available in the literature and demonstrate the multiphoton multiple ionization dynamics of $\mathrm{H}_{2} \mathrm{O}$ when exposed to an intense X-ray pulse. In Sec. IV, we draw conclusions.

\section{COMPUTATIONAL METHODS}

\section{A. Rate equations}

We follow the electronic dynamics based on the PauliMaster equation, in which the time-dependent evolution of the population of an electronic state is described via transition rates. We subsume electronic states under electronic configurations that specify the occupancy (zero, one, or two) of each molecular orbital. For the x-ray dynamics of a single water molecule, we consider zero, single, or double vacancies in each of the five molecular orbitals, i.e., we consider $3^{5}=243$ coupled rate equations. The time evolution of the population $P_{I}(t)$ for an electronic configuration $I$ is given by

$$
\frac{d}{d t} P_{I}(t)=\sum_{I^{\prime} \neq I}\left[\Gamma_{I^{\prime} \rightarrow I} P_{I^{\prime}}(t)-\Gamma_{I \rightarrow I^{\prime}} P_{I}(t)\right]
$$

where the quantities $\Gamma_{I \rightarrow I^{\prime}}$ are the rates for the Auger or fluorescence decay from electronic configuration $I$ to $I^{\prime}$, or the photoionization rates for the ionization process from $I$ to $I^{\prime}$. The photoionization rates are determined as $\Gamma_{I \rightarrow I^{\prime}}=$ $\sigma_{I \rightarrow I^{\prime}} J(t)$ from the instantaneous X-ray flux $J(t)$ at time $t$ and the ionization cross section $\sigma_{I \rightarrow I^{\prime}}$. For simplicity, we solely focus on the electronic radiation damage, i.e., we keep the nuclei fixed. Possible consequences of this approximation will be discussed in Sec. III B.

\section{B. Electronic structure}

The number of electronic configurations that can be visited during the $\mathrm{x}$-ray ionization dynamics of a single water molecule is already quite large $\left(3^{5}=243\right)$ and this number can be much larger for more complex molecules. To model the electronic structure and determine the relevant decay rates and ionization cross sections, it is crucial that the electronic structure model is computationally cheap. We employ the molecular Hartree-Fock-Slater (HFS) method [28] as implemented in the XMOLECULE toolkit [27]. Details of the molecular electronic structure calculation are described in Ref. [27]. Here we summarize the essence of the computational procedure.

For every electronic hole configuration that is relevant for the ionization dynamics, molecular orbitals (MOs) are derived from an HFS self-consistent-field (SCF) calculation. Electronic rearrangements describing charge migration are modeled here by reoptimizing the MOs for every electronic hole configuration visited in a sequence of photoionization, Auger, and fluorescence processes. As basis functions for molecular calculations, we employ numerical atomic orbitals that are obtained from an atomic HFS calculation adjusted for the respective core hole state [27]. These core-hole-adapted numerical atomic orbitals are accurately calculated by the XATOM toolkit [29]. With these basis functions $\left\{\chi_{\mu}(\mathbf{r})\right\}$, the MOs are expanded as

$$
\phi_{a}(\mathbf{r})=\sum_{\mu} C_{\mu a} \chi_{\mu}(\mathbf{r})
$$

where $a$ is the MO index and $\left\{C_{\mu a}\right\}$ are the coefficients optimized in the Roothaan-Hall-type SCF calculation. Here $\mu$ is the basis index, which also contains the atomic index.

\section{Transition rates}

In the following, we describe in detail how molecular transition rates for the Auger, fluorescence, and photoionization processes are determined efficiently in the molecular calculation. Throughout the calculation of transition rates, we employ the independent-electron picture. Specifically, for each transition we use MOs derived from the HFS calculation of the respective initial-state configuration in order to evaluate transitions to all final-state configurations.

Calculating ionization transition rates for molecules requires a description of the molecular continuum wave functions. The evaluation of these electronic continuum wave functions is not trivial (e.g., [30,31]). Instead of using the explicit molecular continuum wave functions, we employ atomic continuum wave functions that are calculated from the same potentials used in the corresponding atomic calculations for generating basis functions. The atomic continuum wave functions are computed by the ХАTOM toolkit [29].

In our scheme, the rate calculation implies a sum over final states and an average over initial states that can be described with the respective initial and final electronic hole configuration.

\section{Photoionization cross sections}

To calculate $\mathrm{x}$-ray photoionization cross sections, we employ the approach described in Refs. [32,33], which is based on the mean-field picture and atomic continuum wave functions. Molecular transition dipole matrix elements are calculated by using the atomic calculations that are used to determine the basis functions. Accordingly, the photoionization cross section for MO $\phi_{a}$ with linearly polarized x-ray light averaged over the molecular orientations is given in the length form by

$$
\sigma_{a}(\omega)=\frac{4}{3} \pi^{2} \alpha \omega \sum_{A}^{\text {atoms }} \sum_{l m} \sum_{d=x, y, z}\left\{\sum_{\substack{\mu \\ \text { on atom } A}} C_{\mu a}^{2}\left|\left\langle\chi_{\mu}|d| \chi_{\epsilon l m}\right\rangle\right|^{2}+2 \sum_{\substack{\mu<\nu \\ \text { on atom } A}} C_{\mu a} C_{v a}\left\langle\chi_{\mu}|d| \chi_{\epsilon l m}\right\rangle\left\langle\chi_{\nu}|d| \chi_{\epsilon l m}\right\rangle\right\},
$$

where $\alpha$ is the fine-structure constant, $\omega$ is the photon energy, and $\left\langle\chi_{\mu}|d| \chi_{\epsilon l m}\right\rangle$ are transition dipole matrix elements involv- ing the basis functions $\chi_{\mu}(\mathbf{r})$ and the energy-normalized atomic continuum wave function $\chi_{\epsilon l m}(\mathbf{r})$ on atom $A$ with orbital 
angular momentum quantum number $l$ and the associated projection quantum number $m$. The photoelectron energy is determined via $\epsilon=\omega+E_{\mathrm{i}}-E_{\mathrm{f}}$, where $E_{\mathrm{i}}$ and $E_{\mathrm{f}}$ are the initial and final bound state energies, respectively. In the present calculations, we estimate the photoelectron energy as $\epsilon=\omega+\epsilon_{a}$, i.e., we employ Koopmans' theorem [34] using the HFS MO energy eigenvalue $\epsilon_{a}$.

The transition dipole matrix elements between basis functions and continuum wave functions, $\left\langle\chi_{\mu}|d| \chi_{\epsilon l m}\right\rangle$, are readily obtained from the atomic HFS calculation using the XATOM toolkit [29]. Specifically, we calculate a set of atomic transition dipole matrix elements at a discrete set of photoelectron energies and interpolate the transition dipole matrix elements in the molecular calculation at the required photoelectron energy.

\section{Auger decay rates}

The Auger decay amplitude for a single Slater determinant with a hole in a spin orbital $\left(c, \sigma_{c}\right)$ to a final Slater determinant with valence vacancies in spin orbitals $\left(a, \sigma_{a}\right)$ and $\left(b, \sigma_{b}\right)$ and an Auger electron in spin continuum $\left(k, \sigma_{k}\right)$ is given by [35]

$$
\begin{aligned}
A_{\mathrm{i} \rightarrow \mathrm{f} k} \sim & \left(\langle a b \mid c k\rangle \delta_{\sigma_{a}, \sigma_{c}} \delta_{\sigma_{b}, \sigma_{k}}-\langle b a \mid c k\rangle \delta_{\sigma_{b}, \sigma_{c}} \delta_{\sigma_{a}, \sigma_{k}}\right) \\
& \times\left\langle\Psi_{\mathrm{i}}\left|c_{c, \sigma_{c}} c_{a, \sigma_{a}}^{\dagger} c_{b, \sigma_{b}}^{\dagger}\right| \Psi_{\mathrm{f}}\right\rangle,
\end{aligned}
$$

where $\Psi_{\mathrm{i}}$ and $\Psi_{\mathrm{f}}$ represent the initial and final Slater determinants without electronic continuum and $c_{a, \sigma_{a}}^{\dagger}\left(c_{a, \sigma_{a}}\right)$ is the creation (annihilation) operator for an electron in the spin orbital $\left(a, \sigma_{a}\right)$. The symbol $\delta_{\sigma_{a}, \sigma_{c}}$ is the Kronecker delta for the respective spin quantum numbers $\sigma_{a}$ and $\sigma_{c}$, and the symbol $\langle a b \mid c k\rangle$ is the two-electron integral given by

$$
\langle a b \mid c k\rangle=\int d^{3} r_{1} \int d^{3} r_{2} \phi_{a}\left(\mathbf{r}_{1}\right) \phi_{b}\left(\mathbf{r}_{2}\right) \frac{1}{\left|\mathbf{r}_{1}-\mathbf{r}_{2}\right|} \phi_{c}\left(\mathbf{r}_{1}\right) \phi_{k}\left(\mathbf{r}_{2}\right),
$$

involving the spatial orbitals $\phi_{a}(\mathbf{r}), \phi_{b}(\mathbf{r})$, and $\phi_{c}(\mathbf{r})$. The function $\phi_{k}(\mathbf{r})$ represents an energy-normalized continuum wave function with the Auger electron energy $\epsilon_{k}=k^{2} / 2=$ $E_{\mathrm{i}}-E_{\mathrm{f}}$.

Employing the averaging over different spin configurations and summing over all continuum channels with energy $\epsilon_{k}$, the Auger transition rates are given by

$$
\begin{aligned}
\Gamma_{a, b \rightarrow c}= & 2 \pi \sum_{k}^{k^{2} / 2=\epsilon_{k}}\left(2-n_{c}\right) N_{a b}\left[\frac{1}{2}|(\langle a b \mid c k\rangle+\langle b a \mid c k\rangle)|^{2}\right. \\
& \left.+\frac{3}{2}|(\langle a b \mid c k\rangle-\langle b a \mid c k\rangle)|^{2}\right]
\end{aligned}
$$

with the statistical factor

$$
N_{a b}= \begin{cases}\frac{n_{a} n_{b}}{4} & \text { if } a \neq b \\ \frac{n_{a}\left(n_{a}-1\right)}{4} & \text { if } a=b\end{cases}
$$

where $n_{a}, n_{b}$, and $n_{c}$ are the occupancies of the spatial orbitals $\phi_{a}, \phi_{b}$, and $\phi_{c}$, respectively. The sum over $k$ in Eq. (6) is meant as a sum over all continuum channels with the energy $\epsilon_{k}$. The two terms inside the square brackets in Eq. (6) can be interpreted as the singlet and triplet contributions to the Auger transition rate. They are weighted by statistical factors for finding the spatial orbital combination $\phi_{a}$ and $\phi_{b}$ being occupied, $N_{a b}$, and the spatial orbital $\phi_{c}$ being vacant, $\left(2-n_{c}\right)$.

To evaluate the Auger transition rates, we employ the socalled one-center approximation [36-41]. This approximation is based on the fact that in most cases the core orbital $\phi_{c}$ is strongly localized on atom $A$ such that the Auger process can be largely understood as an intra-atomic process only involving the parts of the electronic wave functions close to the atom $A$. Accordingly, the molecular continuum wave functions $\phi_{k}(\mathbf{r})$ are approximated with the atomic continuum wave functions $\chi_{\kappa}(\mathbf{r})$, and the continuum energy is given by the atomic Auger energy, $\epsilon_{k}=\epsilon_{\kappa}$, where $\kappa$ is the basis index corresponding to the atom $A$. Further, the two-electron integrals are expanded by using the linear combination of atomic orbitals on the atom $A$, such that they are approximately given as

$$
\langle a b \mid c k\rangle \simeq \sum_{\substack{\mu \nu \lambda \\ \text { on atom } A}} C_{\mu a} C_{\nu b} C_{\lambda c}\langle\mu \nu \mid \lambda \kappa\rangle
$$

To evaluate these quantities, we determine the continuum energy $\epsilon_{\kappa}$ from the atomic orbital energy eigenvalues as $\epsilon_{\kappa}=\epsilon_{\mu}+\epsilon_{\nu}-\epsilon_{\lambda}$, based on the one-center approximation. All these atomic quantities are computed using the XATOM toolkit [29].

Note that, although the evaluation of the molecular Auger rates is based on intra-atomic processes, it does not mean that the molecular Auger transition involves only the local atomic electronic structure. In fact, a molecular valence orbital, which is delocalized over many atoms, may give rise to an Auger process that ionizes not only the atom where the core hole is initially located but also neighboring atoms.

\section{Fluorescence rates}

The transition rate for the fluorescence decay that reoccupies a hole in $\mathrm{MO} \phi_{c}$ with an electron from $\mathrm{MO} \phi_{a}$ is given by

$$
\Gamma_{a \rightarrow c}=\frac{n_{a}\left(2-n_{c}\right)}{2} \frac{4\left(\alpha \omega_{f}\right)^{3}}{3} \sum_{d=x, y, z}\left|\left\langle\phi_{c}|d| \phi_{a}\right\rangle\right|^{2},
$$

where the fluorescence photon energy $\omega_{f}$ is taken as the energy difference between initial and final hole configuration. The quantities $\left\langle\phi_{c}|d| \phi_{a}\right\rangle$ are the transition dipole matrix elements between the MOs $\phi_{a}$ and $\phi_{c}$ with occupation $n_{a}$ and $n_{c}$, respectively. The statistical factor $\frac{n_{a}}{2}\left(2-n_{c}\right)$ arises from the spin configuration averaging. For our evaluation, we approximate the fluorescence energy by the orbital energy difference, i.e., $\omega_{f}=\epsilon_{a}-\epsilon_{c}$.

\section{RESULTS}

\section{A. Molecular Auger decay rates for the first-row hydrides}

Since Auger decay plays an important role for ionization dynamics, we first discuss the accuracy of the Auger rates calculated in this work. For the molecular calculations we employ the minimal basis set plus additional $p$ - and $d$-type basis functions for the first-row elements and an additional $p$-type basis function for hydrogen, as described in Ref. [27]. Our calculated values are compared with Auger transition rates from experimental data and other calculations available in the 
TABLE I. Comparison of calculated total Auger decay rates for the single-core hole $(\mathrm{SCH})$ and double-core hole (DCH) in molecules of the first-row hydride series. Experimental results are also listed if available. Values are given in $10^{-3}$ atomic units.

\begin{tabular}{|c|c|c|c|}
\hline & & $\mathrm{SCH}$ & $\mathrm{DCH}$ \\
\hline $\mathrm{CH}_{4}$ & $\begin{array}{l}\text { Present } \\
\text { Inhester } \text { et al. [42] } \\
\text { Larkins [43] } \\
\text { Kolorenč and Averbukh [44] } \\
\text { Carroll } \text { et al. } \text { [45] } \\
\text { Köppe } \text { et al. [46] }\end{array}$ & $\begin{array}{c}2.3 \\
2.7 \\
3.5 \\
3.1 \\
3.4-3.5^{\mathrm{a}} \\
2.7-3.5^{\mathrm{a}}\end{array}$ & $\begin{array}{r}10.3 \\
9.4\end{array}$ \\
\hline $\mathrm{NH}_{3}$ & $\begin{array}{l}\text { Present } \\
\text { Inhester } \text { et al. } \text { [42] } \\
\text { Larkins [43] } \\
\text { Kolorenč and Averbukh [44] }\end{array}$ & $\begin{array}{l}3.9 \\
3.7 \\
5.2 \\
4.5\end{array}$ & $\begin{array}{l}15.0 \\
12.4\end{array}$ \\
\hline $\mathrm{H}_{2} \mathrm{O}$ & $\begin{array}{l}\text { Present } \\
\text { Inhester } \text { et al. [42] } \\
\text { Larkins [43] } \\
\text { Kolorenč and Averbukh [44] } \\
\text { Carravetta and Ågren [47] } \\
\text { Sankari } \text { et al. [48] }\end{array}$ & $\begin{array}{c}5.7 \\
5.0 \\
6.8 \\
5.4 \\
5.5 \\
5.6-6.0^{\mathrm{a}}\end{array}$ & $\begin{array}{l}20.0 \\
15.4\end{array}$ \\
\hline $\mathrm{HF}$ & $\begin{array}{l}\text { Present } \\
\text { Inhester } \text { et al. [42] } \\
\text { Larkins [43] }\end{array}$ & $\begin{array}{l}7.7 \\
6.5 \\
8.3\end{array}$ & $\begin{array}{l}25.0 \\
19.2\end{array}$ \\
\hline
\end{tabular}

${ }^{\mathrm{a}}$ Experimental results.

literature. Table I lists the total Auger decay rates for a single and double core vacancy in molecules of the first-row hydride series. As can be seen, the values we obtain for the single-corehole $(\mathrm{SCH})$ Auger processes are in good agreement with the other published values for the SCH Auger rates. For the doublecore-hole (DCH) Auger processes, the values we obtain tend to be slightly larger than the previous results. This discrepancy is largely due to the simplified electronic structure model used here.

\section{B. Charge-state distribution of water at high $x$-ray intensity}

The techniques described above provide the photoionization cross sections, fluorescence, and Auger decay rates for all the multiple core and valence hole configurations involved in the $\mathrm{x}$-ray multiphoton multiple ionization dynamics. With these values we integrated the $3^{5}=243$ rate equations of Eq. (1) for the charge-state populations using the fourth-order Runge-Kutta method for a range of pulse parameters. Figure 1 shows the charge-state distribution of an $\mathrm{H}_{2} \mathrm{O}$ molecule after it has been exposed to an intense x-ray pulse with a photon energy of $12 \mathrm{keV}$ and a Gaussian-shaped temporal profile of $100 \mathrm{fs}$ full width at half maximum (FWHM). The fluence, which is defined as the number of incident $\mathrm{X}$-ray photons per unit area, varies from $10^{14}$ to $10^{15} \mathrm{ph} / \mu \mathrm{m}^{2}$. These numbers are close to and larger than the one-photon absorption saturation fluence for the water molecule at $12 \mathrm{keV}$, which is given by the inverse of the total photoionization cross section $\left(=1 / \sigma_{\mathrm{H}_{2} \mathrm{O}} \approx 10^{14} \mathrm{ph} / \mu \mathrm{m}^{2}\right)$. Therefore, the water molecule absorbs more than one photon in this range of fluences, and

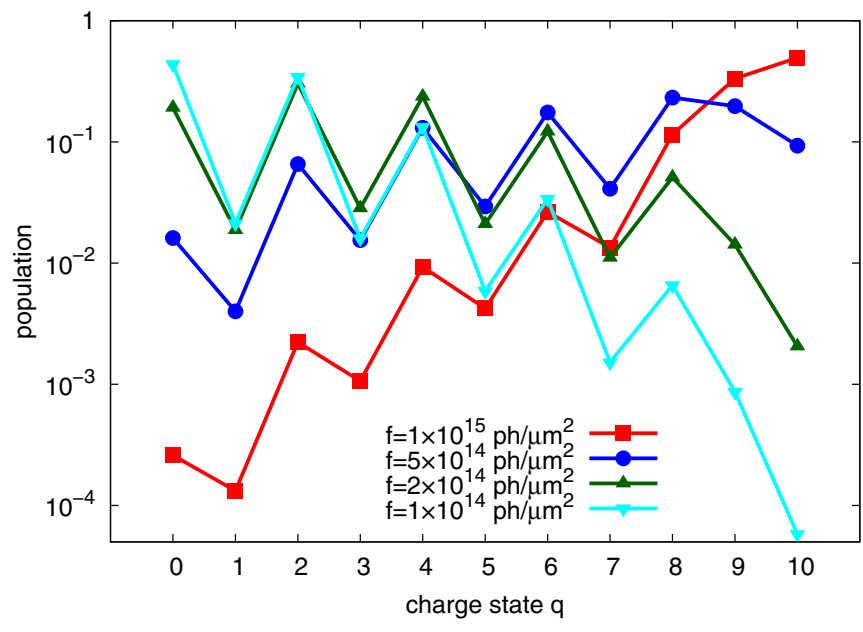

FIG. 1. Charge-state distributions of a water molecule after exposure to an intense $\mathrm{x}$-ray pulse with a Gaussian-shaped pulse envelope (100 fs FWHM) for several fluences.

the sequences of photoionization and Auger decay processes result in high charge states.

As can be seen, for the lower charge states there exists a characteristic alternating pattern such that even charge-state populations are much more strongly populated than odd ones are $[6,7,9]$. This pattern arises from the fact that a core shell ionization is immediately followed by an Auger decay (typically faster than the pulse duration), such that always two charges are produced per absorbed photon, when the pulse duration is long enough. For the fluence of $10^{15} \mathrm{ph} / \mu \mathrm{m}^{2}$, the dominant population is at $q=+10$, where all electrons of the water molecule are stripped off. Production of $q>+8$ demonstrates that molecular effects play a role, because the atomic oxygen has eight electrons and direct photoionization of atomic hydrogen is negligible at $12 \mathrm{keV}$ (the cross section for atomic hydrogen is approximately five orders of magnitude smaller than for atomic oxygen). Within the independent atom model, where all atoms in a molecule are treated individually, the sum of atomic oxygen charge and two hydrogen charges cannot be larger than +8 in the fluence regime considered here.

For the present calculations, we neglect any nuclear motion, although the high degree of ionization may trigger fast nuclear dynamics. The motion of the nuclei may alter the electronic structure in the molecule, which could have impact on the resulting electronic transition rates. We note, however, that in the water molecule Auger decay rates are quite constant with respect to the molecular geometries that appear during the typical lifetime of single or double core vacancies $[49,50]$. Since core ionization on oxygen is dominant here, total photoionization cross section may not be affected by the nuclear motion. Furthermore, for higher ionized states, all valence electrons are dominantly located on the oxygen atom, as will be shown in Fig. 2. Consequently, the position of the remaining protons is less relevant for the further ionization dynamics. For these reasons, we infer that nuclear motion plays a mere significant role for the results presented here. For other molecules the nuclear dynamics may have some implications on the electronic x-ray ionization dynamics and the resulting charges. We will address this aspect in future work [51]. 


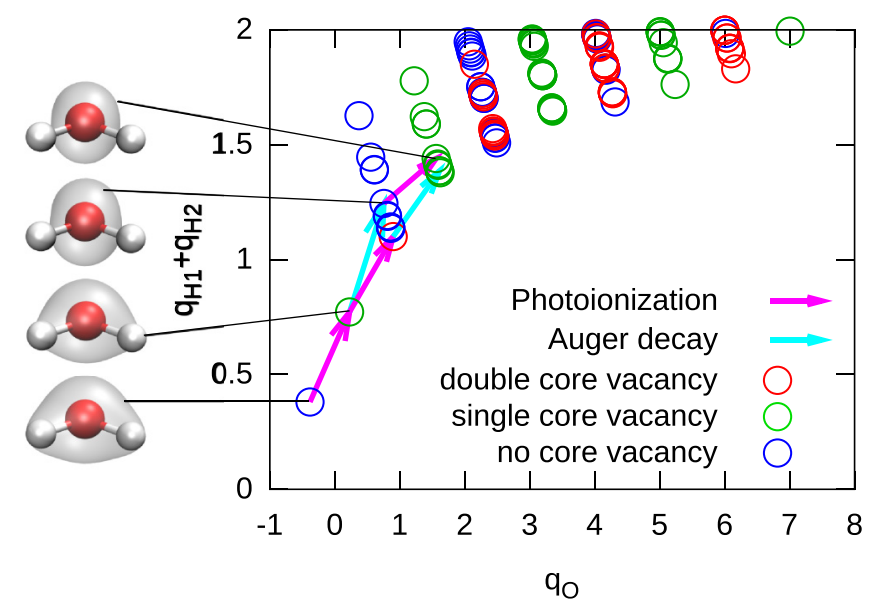

FIG. 2. Partial charge of oxygen vs. partial charge of hydrogens (summed together) during $\mathrm{x}$-ray multiphoton ionization dynamics. For simplicity, only processes initiated by oxygen $K$-shell photoionizations are depicted. The color indicates the single (green), double (red), or no core vacancy (blue). The first steps of two typical sequences are shown with arrows. For a few configurations the molecular electron density is illustrated with an isosurface plot in the left panel.

\section{Charge rearrangement}

Figure 2 shows the partial charges on the hydrogen atoms as a function of the partial charge on the oxygen atom for the electron configurations that can be accessed from a sequence of oxygen $K$-shell photoionization and Auger decay processes. The partial charges are calculated using the Löwdin population analysis. The first ionization steps for two typical sequences are indicated by arrows. Since we consider only oxygen $K$-shell photoionization in the figure, the charging up of hydrogens manifests the degree of charge rearrangement. As one can see, the electron density from the hydrogen atoms is shifted in the first two steps (e.g., core ionization and subsequent Auger decay) largely towards the oxygen atom, such that less than half of an electron is left on each hydrogen atom. With further ionization, this shift towards the oxygen proceeds and eventually all electrons are stripped off the hydrogen atoms. This progressive shift of electronic density is illustrated by isosurface plots for several electronic configurations in the left panel of Fig. 2.

The charge rearrangement can be explained by two molecular effects: orbital relaxation upon sequential ionization and molecular Auger decay. Because of the imbalance of $Z$ among the atomic species in the molecule, photoionization occurs predominantly from the $K$ shell of oxygen. When a positive charge is locally formed on the oxygen, the electrons in the water molecule are pulled towards the oxygen atom. The molecular orbitals relax in every ionization step, such that the bonding orbitals, initially delocalized over all three atoms, get more and more localized on the oxygen atom. Also the molecular orbitals are explicitly involved in our treatment of the molecular Auger decay processes in Eq. (8). Once the Auger decay happens with delocalized valence molecular orbitals, it creates partial charges on the hydrogens, describing the charge rearrangement. These molecular effects

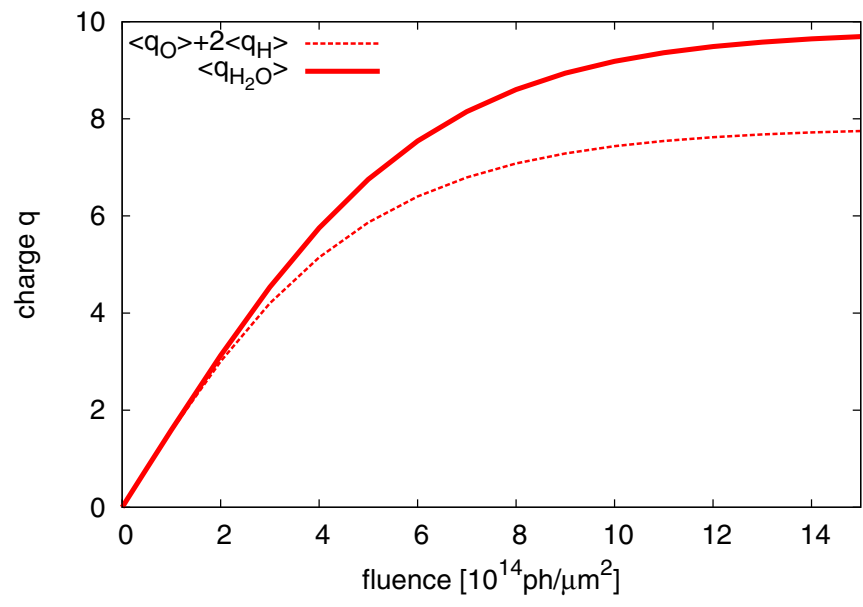

FIG. 3. Total mean charge after the x-ray pulse as a function of fluence for a Gaussian-shaped pulse with duration of $100 \mathrm{fs}$ FWHM and a photon energy of $12 \mathrm{keV}$.

are represented via the molecular orbital approach, which is completely absent in the independent atom model.

\section{Molecular model versus independent atom model}

To quantify the molecular ionization effect, we investigate here the total mean charge of the water molecule after it has been exposed to the intense x-ray pulse. These values are compared to the results from the independent atom model, i.e., the sum of the final mean charges obtained from isolated simulations of the constituent oxygen and hydrogen atoms.

Figure 3 shows the average charge after the x-ray pulse as a function of fluence for the same pulse duration as used in Fig. 1. As can be seen, for low fluences $\left(<2 \times 10^{14} \mathrm{ph} / \mu \mathrm{m}^{2}\right)$ a relatively low ionization yield is observed with a total mean charge $q<+2$. The independent atom calculation yields the same charge as the molecular calculation. At these low fluences (especially lower than the one-photon absorption saturation fluence), one-photon absorption is dominant and the charge rearrangement after a single photoionization event does not increase the total charge of the molecule. In contrast, one can see a clear enhancement of the total charge in the molecular case at higher fluences, where x-ray multiphoton ionization is expected. Repetition of the photoionization and the charge rearrangement keeps providing more electrons from hydrogens to be ionized on the oxygen site. These electrons from hydrogens would not be ionized if they were located at the hydrogens because of their negligible photoionization cross section. At the fluence of $1.5 \times 10^{15} \mathrm{ph} / \mu \mathrm{m}^{2}$, the water molecule is on average fully ionized $(\sim+10)$, whereas with isolated atoms only the oxygen atom is fully ionized, so the molecule reaches on average a total charge of $\sim+8$.

To investigate the molecular ionization enhancement effect for different pulse durations, Fig. 4 shows the total mean charge as a function of the pulse duration at a constant fluence of $10^{15} \mathrm{ph} / \mu \mathrm{m}^{2}$. As can be seen, for larger pulse durations the total mean charge is nearly constant with respect to the pulse duration. With pulse durations $<20 \mathrm{fs}$, the total mean charge decreases, illustrating the frustrated absorption effect $[6,7]$. When the pulse duration approaches $0 \mathrm{fs}$, only the two $K$-shell 


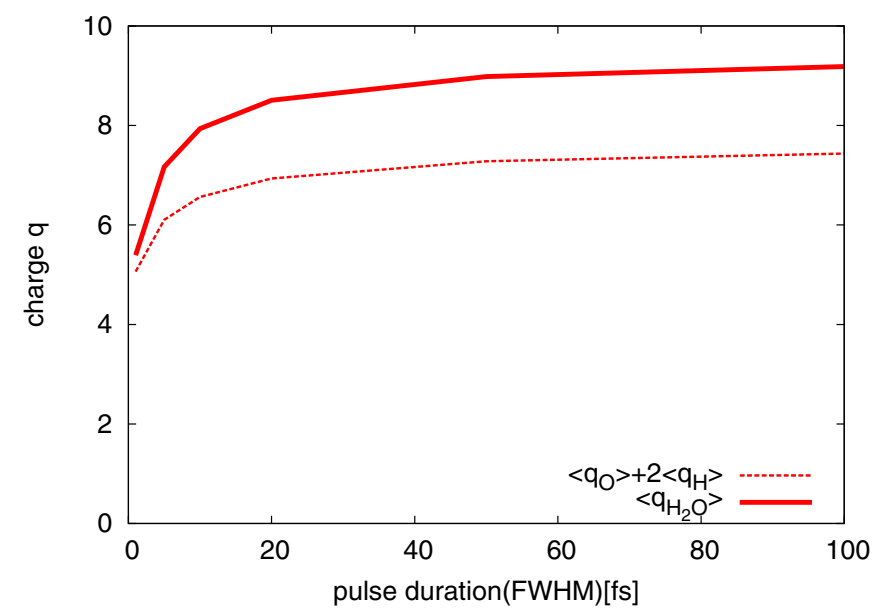

FIG. 4. Total mean charge after the x-ray pulse as a function of pulse duration (FWHM) for a pulse Gaussian-shaped pulse with a photon energy of $12 \mathrm{keV}$ and a fluence of $10^{15} \mathrm{ph} / \mu \mathrm{m}^{2}$.

electrons are ionized and the Auger decay happens after the pulse is over. Thus, within both the independent atom model and the molecular model, the total charge approaches $q=+4$, which is produced by the Auger decay of a double $K$-shell vacancy in the oxygen atom. Therefore, our results show that the molecular ionization enhancement decreases as the pulse duration decreases.

\section{CONCLUSIONS}

In this work, we extend the XMOLECULE toolkit to describe multiphoton multiple ionization dynamics of polyatomic molecules exposed to intense x-ray pulses. The proposed computational method is based on efficient molecular electronic structure calculations, utilizing core-hole-adapted basis functions obtained from XATOM. To efficiently describe the molecular transition rates we employ several approximations, in particular, using atomic continuum wave functions. Orbital optimization and transition rate calculations are conducted for all possible multiple-hole configurations that may be formed during $\mathrm{x}$-ray multiphoton multiple ionization dynamics. To simulate ionization dynamics, we solve a set of coupled rate equations. The proposed method has the potential to be applicable to larger molecules because of its efficient computational procedure.

For a series of polyatomic molecules, the first row hydrides, we present molecular Auger rate calculations, which show good agreement with literature values. For a water molecule, we investigate the molecular effects on the $\mathrm{x}$-ray multiphoton ionization dynamics. For high x-ray fluences, we show that sequences of photoionization and charge rearrangement may lead to enhancement of molecular ionization, since electrons are shifted from low- $Z$ atoms, where photoionization is less likely, to high- $Z$ atoms, where photoionization is more likely, and then those electrons become available for subsequent photoionization events on high- $Z$ atoms. For molecules containing heavier elements with electron-rich neighboring constituents, this molecular ionization enhancement effect can be much more dramatic. Our work in this direction will be reported elsewhere [26].

For the prospective single molecule imaging experiments at XFELs, knowledge of the radiation damage during the pulse exposure is essential. The fact that in molecules different charge-state distributions are generated and higher total charges are reached as compared to what one would expect for isolated atoms has important consequences for the estimation of radiation damage in these XFEL experiments. These molecular effects in x-ray multiphoton ionization dynamics have not been rigorously considered in the theoretical modeling of any sample damage. The computational scheme presented here may support future investigations of $\mathrm{x}$-ray radiation damage at the molecular electronic structure level.

\section{ACKNOWLEDGMENTS}

We thank Oriol Vendrell, Artem Rudenko, and Daniel Rolles for helpful discussions. This work has been supported by the excellence cluster "The Hamburg Centre for Ultrafast Imaging-Structure, Dynamics and Control of Matter at the Atomic Scale" of the Deutsche Forschungsgemeinschaft. Yajiang Hao is supported by the National Natural Science Foundation of China (Grant No. 11004007) and the Fundamental Research Funds for the Central Universities of China.
[1] B. W. J. McNeil and N. R. Thompson, X-ray free-electron lasers, Nature Photon. 4, 814 (2010).

[2] J. P. Marangos, Introduction to the new science with x-ray free electron lasers, Contemp. Phys. 52, 551 (2011).

[3] I. Schlichting and J. Miao, Emerging opportunities in structural biology with $\mathrm{x}$-ray free-electron lasers, Curr. Opin. Struct. Biol. 22, 613 (2012).

[4] R. Neutze, R. Wouts, D. van der Spoel, E. Weckert, and J. Hajdu, Potential for biomolecular imaging with femtosecond X-ray pulses, Nature (London) 406, 752 (2000).

[5] H. N. Chapman, A. Barty, M. J. Bogan, S. Boutet, M. Frank, S. P. Hau-Riege, S. Marchesini, B. W. Woods, S. Bajt, H. Benner, R. A. London, E. Ploenjes, M. Kuhlmann, R. Treusch, S. Duesterer, T. Tschentscher, J. R. Schneider, E. Spiller, T. Moeller, C.
Bostedt, M. Hoener, D. A. Shapiro, K. O. Hodgson, D. Van der Spoel, F. Burmeister, M. Bergh, C. Caleman, G. Huldt, M. M. Seibert, F. R. N. C. Maia, R. W. Lee, A. Szoeke, N. Timneanu, and J. Hajdu, Femtosecond diffractive imaging with a soft-X-ray free-electron laser, Nature Phys. 2, 839 (2006).

[6] L. Young, E. P. Kanter, B. Kraessig, Y. Li, A. M. March, S. T. Pratt, R. Santra, S. H. Southworth, N. Rohringer, L. F. DiMauro, G. Doumy, C. A. Roedig, N. Berrah, L. Fang, M. Hoener, P. H. Bucksbaum, J. P. Cryan, S. Ghimire, J. M. Glownia, D. A. Reis, J. D. Bozek, C. Bostedt, and M. Messerschmidt, Femtosecond electronic response of atoms to ultra-intense X-rays, Nature (London) 466, 56 (2010).

[7] M. Hoener, L. Fang, O. Kornilov, O. Gessner, S. T. Pratt, M. Gühr, E. P. Kanter, C. Blaga, C. Bostedt, J. D. Bozek, 
P. H. Bucksbaum, C. Buth, M. Chen, R. Coffee, J. Cryan, L. F. DiMauro, M. Glownia, E. Hosler, E. Kukk, S. R. Leone, B. McFarland, M. Messerschmidt, B. Murphy, V. Petrovic, D. Rolles, and N. Berrah, Ultraintense X-Ray Induced Ionization, Dissociation, and Frustrated Absorption in Molecular Nitrogen, Phys. Rev. Lett. 104, 253002 (2010).

[8] L. Fang, M. Hoener, O. Gessner, F. Tarantelli, S. T. Pratt, O. Kornilov, C. Buth, M. Gühr, E. P. Kanter, C. Bostedt, J. D. Bozek, P. H. Bucksbaum, M. Chen, R. Coffee, J. Cryan, M. Glownia, E. Kukk, S. R. Leone, and N. Berrah, Double CoreHole Production in $\mathrm{N}_{2}$ : Beating the Auger Clock, Phys. Rev. Lett. 105, 083005 (2010).

[9] G. Doumy, C. Roedig, S.-K. Son, C. I. Blaga, A. D. DiChiara, R. Santra, N. Berrah, C. Bostedt, J. D. Bozek, P. H. Bucksbaum, J. P. Cryan, L. Fang, S. Ghimire, J. M. Glownia, M. Hoener, E. P. Kanter, B. Krässig, M. Kuebel, M. Messerschmidt, G. G. Paulus, D. A. Reis, N. Rohringer, L. Young, P. Agostini, and L. F. DiMauro, Nonlinear Atomic Response to Intense Ultrashort X rays, Phys. Rev. Lett. 106, 083002 (2011).

[10] B. Rudek, S.-K. Son, L. Foucar, S. W. Epp, B. Erk, R. Hartmann, M. Adolph, R. Andritschke, A. Aquila, N. Berrah, C. Bostedt, J. Bozek, N. Coppola, F. Filsinger, H. Gorke, T. Gorkhover, H. Graafsma, L. Gumprecht, A. Hartmann, G. Hauser, S. Herrmann, H. Hirsemann, P. Holl, A. Hömke, L. Journel, C. Kaiser, N. Kimmel, F. Krasniqi, K.-U. Kühnel, M. Matysek, M. Messerschmidt, D. Miesner, T. Möller, R. Moshammer, K. Nagaya, B. Nilsson, G. Potdevin, D. Pietschner, C. Reich, D. Rupp, G. Schaller, I. Schlichting, C. Schmidt, F. Schopper, S. Schorb, C.-D. Schröter, J. Schulz, M. Simon, H. Soltau, L. Strüder, K. Ueda, G. Weidenspointner, R. Santra, J. Ullrich, A. Rudenko, and D. Rolles, Ultra-efficient ionization of heavy atoms by intense x-ray free-electron laser pulses, Nature Photon. 6, 858 (2012).

[11] B. Rudek, D. Rolles, S.-K. Son, L. Foucar, B. Erk, S. Epp, R. Boll, D. Anielski, C. Bostedt, S. Schorb, R. Coffee, J. Bozek, S. Trippel, T. Marchenko, M. Simon, L. Christensen, S. De, S. I. Wada, K. Ueda, I. Schlichting, R. Santra, J. Ullrich, and A. Rudenko, Resonance-enhanced multiple ionization of krypton at an X-ray free-electron laser, Phys. Rev. A 87, 023413 (2013).

[12] H. Fukuzawa, S.-K. Son, K. Motomura, S. Mondal, K. Nagaya, S. Wada, X.-J. Liu, R. Feifel, T. Tachibana, Y. Ito, M. Kimura, T. Sakai, K. Matsunami, H. Hayashita, J. Kajikawa, P. Johnsson, M. Siano, E. Kukk, B. Rudek, B. Erk, L. Foucar, E. Robert, C. Miron, K. Tono, Y. Inubushi, T. Hatsui, M. Yabashi, M. Yao, R. Santra, and K. Ueda, Deep Inner-Shell Multiphoton Ionization by Intense X-ray Free-Electron Laser Pulses, Phys. Rev. Lett. 110, 173005 (2013).

[13] K. Motomura, H. Fukuzawa, S.-K. Son, S. Mondal, T. Tachibana, Y. Ito, M. Kimura, K. Nagaya, T. Sakai, K. Matsunami, S. Wada, H. Hayashita, J. Kajikawa, X.-J. Liu, R. Feifel, P. Johnsson, M. Siano, E. Kukk, B. Rudek, B. Erk, L. Foucar, E. Robert, C. Miron, K. Tono, Y. Inubushi, T. Hatsui, M. Yabashi, M. Yao, R. Santra, and K. Ueda, Sequential multiphoton multiple ionization of atomic argon and xenon irradiated by $\mathrm{x}$-ray free-electron laser pulses at SACLA, J. Phys. B 46, 164024 (2013).

[14] M. Bergh, N. Tîmneanu, and D. van der Spoel, Model for the dynamics of a water cluster in an x-ray free electron laser beam, Phys. Rev. E 70, 051904 (2004).
[15] Z. Jurek, G. Faigel, and M. Tegze, Dynamics in a cluster under the influence of intense femtosecond hard x-ray pulses, Eur. Phys. J. D 29, 217 (2004).

[16] S. P. Hau-Riege, Nonequilibrium electron dynamics in materials driven by high-intensity x-ray pulses, Phys. Rev. E 87, 053102 (2013).

[17] S. P. Hau-Riege, R. A. London, and A. Szoke, Dynamics of biological molecules irradiated by short x-ray pulses, Phys. Rev. E 69, 051906 (2004).

[18] B. Ziaja, Z. Jurek, N. Medvedev, V. Saxena, S.-K. Son, and R. Santra, Towards realistic simulations of macromolecules irradiated under the conditions of coherent diffraction imaging with an x-ray free-electron laser, Photonics 2, 256 (2015)

[19] C. Buth, J. Liu, M. H. Chen, J. P. Cryan, L. Fang, J. M. Glownia, M. Hoener, R. N. Coffee, and N. Berrah, Ultrafast absorption of intense $\mathrm{x}$ rays by nitrogen molecules, J. Chem. Phys. 136, 214310 (2012).

[20] B. Erk, D. Rolles, L. Foucar, B. Rudek, S. W. Epp, M. Cryle, C. Bostedt, S. Schorb, J. Bozek, A. Rouzee, A. Hundertmark, T. Marchenko, M. Simon, F. Filsinger, L. Christensen, S. De, S. Trippel, J. Küpper, H. Stapelfeldt, S. I. Wada, K. Ueda, M. Swiggers, M. Messerschmidt, C. D. Schröter, R. Moshammer, I. Schlichting, J. Ullrich, and A. Rudenko, Ultrafast Charge Rearrangement and Nuclear Dynamics upon Inner-Shell Multiple Ionization of Small Polyatomic Molecules, Phys. Rev. Lett. 110, 053003 (2013).

[21] B. Erk, D. Rolles, L. Foucar, B. Rudek, S. W. Epp, M. Cryle, C. Bostedt, S. Schorb, J. Bozek, A. Rouzee, A. Hundertmark, T. Marchenko, M. Simon, F. Filsinger, L. Christensen, S. De, S. Trippel, J. Küpper, H. Stapelfeldt, S.I. Wada, K. Ueda, M. Swiggers, M. Messerschmidt, C. D. Schröter, R. Moshammer, I. Schlichting, J. Ullrich, and A. Rudenko, Inner-shell multiple ionization of polyatomic molecules with an intense $\mathrm{x}$-ray freeelectron laser studied by coincident ion momentum imaging, J. Phys. B 46, 164031 (2013).

[22] B. Erk, R. Boll, S. Trippel, D. Anielski, L. Foucar, B. Rudek, S. W. Epp, R. Coffee, S. Carron, S. Schorb, K. R. Ferguson, M. Swiggers, J. D. Bozek, M. Simon, T. Marchenko, J. Küpper, I. Schlichting, J. Ullrich, C. Bostedt, D. Rolles, and A. Rudenko, Imaging charge transfer in iodomethane upon $\mathrm{X}$-ray photoabsorption, Science 345, 288 (2014).

[23] K. Schnorr, A. Senftleben, M. Kurka, A. Rudenko, G. Schmid, T. Pfeifer, K. Meyer, M. Kübel, M. F. Kling, Y. H. Jiang, R. Treusch, S. Düsterer, B. Siemer, M. Wöstmann, H. Zacharias, R. Mitzner, T. J. M. Zouros, J. Ullrich, C. D. Schröter, and R. Moshammer, Electron Rearrangement Dynamics in Dissociating $\mathrm{I}_{2}^{n+1}$ Molecules Accessed by Extreme Ultraviolet Pump-Probe Experiments, Phys. Rev. Lett. 113, 073001 (2014).

[24] B. F. Murphy, T. Osipov, Z. Jurek, L. Fang, S.-K. Son, M. Mucke, J. H. D. Eland, V. Zhaunerchyk, R. Feifel, L. Avaldi, P. Bolognesi, C. Bostedt, J. D. Bozek, J. Grilj, M. Gühr, L. J. Frasinski, J. Glownia, D. T. Ha, K. Hoffmann, E. Kukk, B. K. McFarland, C. Miron, E. Sistrunk, R. J. Squibb, K. Ueda, R. Santra, and N. Berrah, Femtosecond $x$-ray-induced explosion of C60 at extreme intensity, Nat. Commun. 5, 4281 (2014).

[25] J.-C. Liu, N. Berrah, L. S. Cederbaum, J. P. Cryan, J. M. Glownia, K. J. Schafer, and C. Buth, Rate equations for nitrogen molecules in ultrashort and intense x-ray pulses, J. Phys. B 49, 075602 (2016). 
[26] A. Rudenko et al. (unpublished).

[27] Y. Hao, L. Inhester, K. Hanasaki, S.-K. Son, and R. Santra, Efficient electronic structure calculation for molecular ionization dynamics at high x-ray intensity, Struct. Dyn. 2, 041707 (2015).

[28] J. C. Slater, A simplification of the Hartree-Fock method, Phys. Rev. 81, 385 (1951).

[29] S.-K. Son, L. Young, and R. Santra, Impact of hollow-atom formation on coherent $\mathrm{X}$-ray scattering at high intensity, Phys. Rev. A 83, 033402 (2011).

[30] M. Brosolo and P. Decleva, Variational approach to continuum orbitals in a spline basis: An application to $\mathrm{H}^{2+}$ photoionization, Chem. Phys. 159, 185 (1992).

[31] Ph. V. Demekhin, A. Ehresmann, and V. L. Sukhorukov, Single center method: A computational tool for ionization and electronic excitation studies of molecules, J. Chem. Phys. 134, 024113 (2011).

[32] U. Gelius and K. Siegbahn, ESCA studies of molecular core and valence levels in the gas phase, Faraday Discuss. 54, 257 (1972).

[33] Jan-Tsyu J. H. and F. O. Ellison, ESCA: A theoretical intensity model based on the plane-wave approximation, J. Electron Spectrosc. Relat. Phenom. 4, 233 (1974).

[34] T. Koopmans, Über die Zuordnung von Wellenfunktionen und Eigenwerten zu den einzelnen Elektronen eines Atoms, Physica 1, 104 (1934).

[35] R. Manne and H. Ågren, Auger transition amplitudes from general many-electron wavefunctions, Chem. Phys. 93, 201 (1985).

[36] D. L. Walters and C. P. Bhalla, Nonrelativistic K-shell auger rates and matrix elements for $4 \leqslant Z \leqslant 54$, At. Data Nucl. Data Tables 3, 301 (1971).

[37] H. Siegbahn, L. Asplund, and P. Kelfve, The Auger electron spectrum of water vapour, Chem. Phys. Lett. 35, 330 (1975).

[38] M. H. Chen, F. P. Larkins, and B. Crasemann, Auger and CosterKronig radial matrix elements for atomic numbers $6 \leqslant Z \leqslant 92$, At. Data Nucl. Data Tables 45, 1 (1990).

[39] E. Z. Chelkowska and F. P. Larkins, Auger spectroscopy for molecules: Tables of matrix elements for transition-rate calculations corresponding to an s-, p-, or d-type initial hole, At. Data Nucl. Data Tables 49, 121 (1991).

[40] M. Coville and T. D. Thomas, Molecular effects on inner-shell lifetimes: Possible test of the one-center model of auger decay, Phys. Rev. A 43, 6053 (1991).

[41] R. Fink, Theoretical autoionization spectra of $1 s \rightarrow \pi^{*}$ excited $\mathrm{N}_{2}$ and $\mathrm{N}_{2} \mathrm{O}$, J. Electron Spectrosc. Relat. Phenom. 76, 295 (1995).

[42] L. Inhester, G. Groenhof, and H. Grubmüller, Core hole screening and decay rates of double core ionized first row hydrides, J. Chem. Phys. 138, 164304 (2013).

[43] F. P. Larkins, Influence of core hole screening on molecular Auger rates and inner-shell lifetimes, J. Electron Spectrosc. Relat. Phenom. 67, 159 (1994).

[44] P. Kolorenč and V. Averbukh, K-shell Auger lifetime variation in doubly ionized Ne and first row hydrides, J. Chem. Phys. 135, 134314 (2011).

[45] T. X. Carroll, N. Berrah, J. Bozek, J. Hahne, E. Kukk, L. J. Sæthre, and T. D. Thomas, Carbon $1 s$ photoelectron spectrum of methane: Vibrational excitation and core-hole lifetime, Phys. Rev. A 59, 3386 (1999).

[46] H. M. Köppe, B. S. Itchkawitz, A. L. D. Kilcoyne, J. Feldhaus, B. Kempgens, A. Kivimäki, M. Neeb, and A. M. Bradshaw, High-resolution C $1 s$ photoelectron spectra of methane, Phys. Rev. A 53, 4120 (1996).

[47] V. Carravetta and H. Ågren, Stieltjes imaging method for molecular auger transition rates: Application to the auger spectrum of water, Phys. Rev. A 35, 1022 (1987).

[48] R. Sankari, M. Ehara, H. Nakatsuji, Y. Senba, K. Hosokawa, H. Yoshida, A. De Fanis, Y. Tamenori, S. Aksela, and K. Ueda, Vibrationally resolved $\mathrm{O}$ 1s photoelectron spectrum of water, Chem. Phys. Lett. 380, 647 (2003).

[49] H. Ågren, A. Cesar, and V. Carravetta, On the constant resonance width approximation for core electron ionization, Chem. Phys. Lett. 139, 145 (1987).

[50] L. Inhester, C. F. Burmeister, G. Groenhof, and H. Grubmüller, Auger spectrum of a water molecule after single and double core ionization, J. Chem. Phys. 136, 144304 (2012).

[51] K. Hanasaki et al. (unpublished). 